\title{
Chair Fans as Energy Efficient Strategy to Aid Localized Ventilation
}

\author{
Carine Habchi ${ }^{1}$, Kamel Ghali ${ }^{2}$, and Nesreen Ghaddar $^{3}$ \\ 1,2,3 Department of Mechanical Engineering \\ American University of Beirut \\ P.O. Box 11-0236, Beirut 1107-2020, Lebanon, e-mail: chh05@mail.aub.edu, ka04@aub.edu.lb, farah@aub.edu.lb
}

\begin{abstract}
Nowadays, the air conditioning industry is deviating from the conventional mixing ventilation technique towards localized ventilation. Displacement ventilation and ceiling personalized ventilation are localized systems presenting a high potential in insuring good indoor air quality while reducing the energy consumption compared to mixing ventilation. In this work it is proposed to enhance their performance by assisting them with chair fans controlling the behavior of occupants' convective plumes.

Computational fluid dynamics models were developed to simulate office spaces ventilated by displacement ventilation and ceiling personalized ventilation equipped with chair fans. The Lagrangian technique was adopted to track particle trajectories to determine particle behavior after generation from occupant respiratory activity. A parametric study was conducted to assess the effect of chair fan flow rate on each system performance in terms of indoor air quality.

Recommendations were given to reduce cross-infection between occupants for both types of localized systems studied with reduced energy consumption. The total chair fans flow rate was optimized for both cases to insure acceptable indoor air quality resulting in significant energy savings. It was found that the optimal total chair fans flow rate per occupant was approximately $14 \mathrm{~L} / \mathrm{s}$ when assisting displacement ventilation while it was 10 $\mathrm{L} / \mathrm{s}$ when aiding ceiling personalized ventilation.
\end{abstract}

\section{Keywords}

Thermal plumes, chair fans, displacement ventilation, ceiling personalized ventilation, energy consumption

\section{Introduction}

People spend more than $80 \%$ of their time in indoor spaces $[1,2]$. Therefore, the indoor environment should be healthy and comfortable. Occupants constitute one of the main sources of contaminants' release especially through the respiratory activities [3,4]. Disease transmission between occupants represents a threat to their health and should be avoided.
In particular, offices are becoming very crowded with occupants under the constraints of reducing the energy consumption and efficient use of the space [5]. The compromise between insuring a high level of indoor air quality (IAQ) and reducing the energy consumption challenges the design of the ventilation system adopted. Therefore new engineering strategies should be implemented to ventilation systems.

Recently, localized air conditioning has gained popularity as it locally provides the occupants needs minimizing energy costs while maintaining good IAQ [6]. Two main types of localized ventilation are present in the heating ventilation and air conditioning (HVAC) industry: vertical and horizontal localized ventilation where the space is divided into zones in the vertical and horizontal directions respectively. The most popular vertical localized system is displacement ventilation (DV) known for its effectiveness in providing high IAQ at a lower energy cost and widely used in office buildings [7]. Unlike conventional air conditioning systems, DV systems turn the air distribution upside down by supplying fresh air near the floor level at a low velocity of less than $0.2 \mathrm{~m} / \mathrm{s}$ and temperature greater than $18{ }^{\circ} \mathrm{C}$ to avoid thermal draft to occupants in the lower zone. The relatively high DV supply temperature compared to conventional mixing ventilation (MV) limits its applicability to cooling loads lower than $40 \mathrm{~W} / \mathrm{m}^{2}$. Therefore, for higher loads other localized systems should be used.

Horizontal localized systems dividing the space horizontally into microclimates around the occupants surrounded by a macroclimate have captured the attention of many researchers. In fact, localized airflows lead to temperature and particle concentration segregations with low air mixing between the microclimates and macroclimate which allow controlling the transmission of contaminated particles. A recent type of horizontal localized flows consist on ceiling 

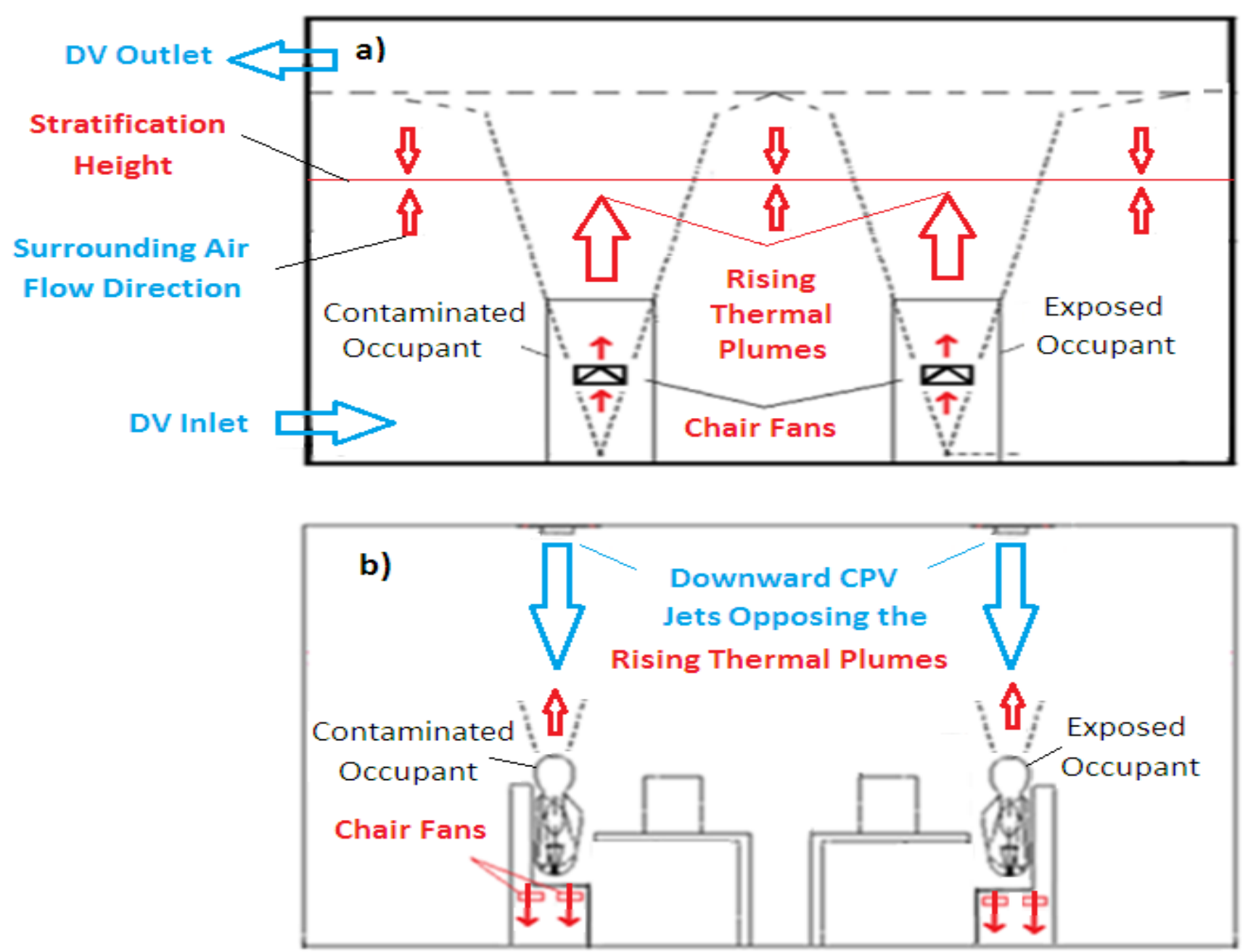

Fig. 1: Chair fans operation for controlling the rising thermal plumes in: a) displacement ventilation system (DV); b) ceiling personalized ventilation system (CPV).

personalized ventilation (CPV) systems which are practical to use since they don't require additional installation cost and can be retrofitted to spaces ventilated by mixed ventilation (MV) systems of ceiling supply type [8].

Melikov [9] underlined the efficiency of controlling airflow interactions in the micro-environment of the human body for improved local air quality and occupant comfort. For this reason, in the current work it is proposed to aid localized ventilation by chair fans $(\mathrm{CF})$.

This work aims towards investigating cross-contamination in office spaces in both vertical localizing DV and horizontal localizing CPV systems (Figure 1). Implementing new engineering strategies to the studied ventilation systems is expected to enhance their performance leading to acceptable IAQ with lower energy consumption.

Computational fluid dynamics (CFD) models were developed to simulate behavior of particles generated by respiratory activities in offices ventilated by DV and
CPV systems. The performance of CF equipped with DV and CPV systems was investigated and optimized. The chair fan flow rate was varied to insure high level of IAQ with reduced energy consumption for both systems.

\section{Methods}

A detailed CFD model that can accurately predict the entrainment of air by the rising plumes and their interaction with the jets of the CF was developed and the Lagrangian technique was used to track particle trajectories. The developed CFD model was used to investigate the effect of the CF operation on DV and $\mathrm{CPV}$ performance in respect with reduced crosscontamination between occupants.

\section{A. CFD Model Features}

To accurately simulate the ventilated space, the CFD model should capture the variable physics affecting the air flow behavior involving turbulence, buoyancy effects, and formation of surfaces boundary layers. The velocity, thermal, and concentration fields were computed using 
the commercial software ANSYS Fluent. The generated grid was constituted by tetrahedral unstructured elements. The Eulerian approach was adopted to simulate the room air because it can be modeled as a continuous fluid [10]. ANSYS Fluent provides a numerical solution for the Navier-Stokes equations by transforming those to algebraic equations. Variable discretization schemes are offered by ANSYS Fluent software and different options for turbulence modeling are provided [11]. The turbulence kinetic energy, dissipation rate, and momentum equations, were discretized by a second-order upwind scheme. The pressure equation was solved by the "PRESTO!" scheme and the coupling between the velocity and pressure fields was performed using the SIMPLE algorithm to [11]. The Realizable k- $\varepsilon$ model was adopted for turbulence modeling as it is accurate in predicting the spread rate of round and planar round jets and convenient in modeling air recirculation [11]. The incompressible ideal gas law was used to consider buoyant plumes [11].

A tetrahedral unstructured mesh was generated and a combination of face sizing and surface inflation was performed for mesh refinement and resolution of the surfaces' boundary layer. Furthermore, enhanced wall treatment was selected for better adaptation of the turbulence modeling near surfaces [11]. This treatment allowed switching between the two-layer model and the enhanced wall function depending on the grid size.

A grid independence test was conducted for proper selection of the mesh elements' number compromising between accuracy and computational time cost. Numerical convergence was judged to be reached when scaled residuals became lower than $10^{-5}$.

\section{B. Modeling of Particle Transport}

The Lagrangian tracking technique was selected to predict the behavior of generated particles within the space. The Lagrangian approach tracks particles' path via the trajectory method which consists on treating the particle dynamics by the second law of newton $[12,13]$. Thus particle motion can be determined by involving the different forces acting on the particle (drag force, lift force, gravitational force...)

In order to investigate the effect of local turbulence intensities on the path of particles, the discrete random walk model (DRW) was adopted. DRW is based on a stochastic approach for modeling the effect of velocity fluctuations on particle trajectories [11,14]. For this reason, the number of tracked particle trajectories should be high enough to insure statistically stable results $[13,15]$. The particle source in-cell (PSI-C) scheme was used to correlate between particle concentration and trajectories within each computational cell $[16,17]$. It was found that 200,000 particle trajectories allowed obtaining a stable solution for the concentration field [17].

\section{Boundary Conditions}

Accurate CFD predictions of the velocity, thermal and concentration fields require appropriate boundary conditions. At inlets, velocity magnitude, direction, and turbulent intensity were specified for modeling of the velocity field. At exhausts, the pressure outlet condition with zero gage pressure was selected. On the other hand, at fan inlets, the "fan" boundary condition was adopted and the pressure drop was determined for fans' jets modeling [18]. For thermal field prediction, heat fluxes were determined at surfaces.

For concentration field modeling, the characteristics of particle generation resulting from normal breathing were used [19]. As in indoor environments airflow velocities are much lower than the ones needed for particle detachment [20], particle re-suspension can be neglected. In order to model particle deposition, the "trap" treatment of the Lagrangian technique was selected at walls [17]. At the outlet, the "escape" treatment was adopted to end the particle trajectory.

\section{Problem Description}

The aim of this work is to investigate the ability of DV and CPV systems in reducing cross-infection between occupants in typical office spaces. Two-station office spaces occupied by two persons were considered. One occupant generated particles simulating the infected person while the other one represented the exposed person. In order to enhance the performance of both systems it was proposed to assist them with CF.

The mode of operation of the CF should be selected in correspondence with the type of ventilation. In fact, CF assistance targets the proper control of the convective human plumes. In DV systems air motion is triggered by buoyancy effects creating vertical upward flow in the occupied zone. In this case, rising thermal plumes play a positive role in carrying contaminants generated by the infected person upward to be exhausted at the ceiling level [21]. Therefore, when assisted with DV, the CF should be operated to strengthen the rising thermal plumes by blowing air upwards thus favoring the removal of contaminants and exposed occupant protection from disease transmission (see Fig. 1). On the other hand, in CPV systems, the occupant upward convective plumes oppose the delivery of the downward CPV jet (see Fig. 1). Hence, when aiding CPV the CF should suppress the occupant plumes by sucking them downward to facilitate the propagation of the downward CPV jet (Fig. 1).

Using the proper direction of the $\mathrm{CF}$ jets depending on the ventilation configuration, the $\mathrm{CF}$ flow rate was optimized for reduced cross-contamination between the occupants.

\section{Results and Discussions}

\section{A. Assisting DV by $C F$}

The purpose of assisting DV with CF was to increase the strength of the thermal plumes. This increase played a double positive role. On one hand, increasing the infected 
plume strength enhanced its effectiveness in the removal of contaminated particles. On the other hand, it improved the protective effectiveness of the exposed plume which constituted a barrier to particle diffusion to the exposed plume and blew relatively clean air to the occupant's breathing zone. The total CF flow rate per occupant was varied from 0 to $20 \mathrm{~L} / \mathrm{s}$ for optimization. Figure 2 represents the effect of the total $\mathrm{CF}$ flow rate per occupant on: a) the intake fraction of $1 \mu \mathrm{m}$ particles at the exposed occupant breathing level for different DV supply flow rates; and b) the variation with height of the average normalized concentration of $1 \mu \mathrm{m}$ particles within the surrounding air for a DV supply flow rate of $80 \mathrm{~L} / \mathrm{s}$.

The intake fraction represents the ratio of the contaminant concentration in the air inhaled by the exposed person to the contaminant concentration in the air exhaled by the infected occupant. The intake fraction is an indication of the air quality at the breathing level of the healthy occupant. On the other hand, the normalized concentration within the surrounding air is the ratio of the contaminant concentration in the surrounding air to the contaminant concentration in the exhaled air of the infected occupant. The observation of the variation of this variable with height illustrates the concentration stratification created by the DV system.

With the increment of the CF flow rate, the strength of the contaminated flows increased (Fig. 1) and the sucked clean air reached higher levels within the exposed plume (see Fig. 1). Therefore, with the increase of the CF flow rate the contaminants' transport by the infected plume was strengthened and particle diffusion from the surrounding towards the healthy occupant was reduced which explains the decrease of the intake fraction with the operation of $\mathrm{CF}$ compared to the standalone DV system (Fig. 2a). Nevertheless, plume strengthening is accompanied with a reduction of the stratification height (Fig. 2b) defined as the level at which the summation of plumes' flow rates equals the DV supply flow rate. In fact, the stratification height separates between the contaminated and clean zones as the surrounding air flow is downward above this height while the airflow is upward below it due to the mass conservation principle (Fig.1). Therefore, the recirculation of contaminants towards the occupied zone increased with the decrease in the level of the stratification height (Fig. $2 b)$. This has a bad effect on the air quality at the breathing level of the occupants.

Hence, the positive role played by the CF increasing the plumes strength and blowing relatively clean air to the breathing level is opposed by the increase of contaminant transport downward towards the occupied zone. This explains the presence of an optimal CF flow rate for which the positive effect created by the $\mathrm{CF}$ overwhelms the negative effect reducing significantly the crosscontamination between the occupants. For a fixed DV supply flow rate, the intake fraction decreased as the total $\mathrm{CF}$ flow rate per occupant increased from 0 to approximately $14 \mathrm{~L} / \mathrm{s}$. On the other hand, for larger $\mathrm{CF}$ flow rates, the decrease in the level of the stratification height degraded the performance of the CF increasing the intake fraction at the breathing level.
The effectiveness of CF operation increased with small DV flow rates as shown in Figure 2a. In, fact assisting $\mathrm{DV}$ by $\mathrm{CF}$ reduces the difference in intake fractions obtained for different DV supply flow rates compared to the large differences observed for the case of standalone DV system.

When operated at the optimal flow rate the $\mathrm{CF}$ assisting the DV system allows to obtain equivalent IAQ performance of the standalone DV system at much smaller DV supply flow rate resulting in considerable energy savings.

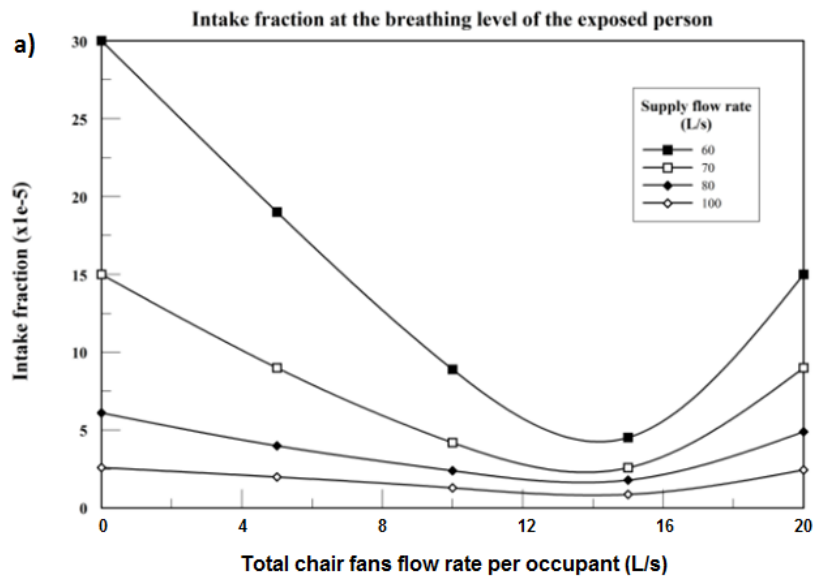

b) Average normalized concentration within the surrounding air

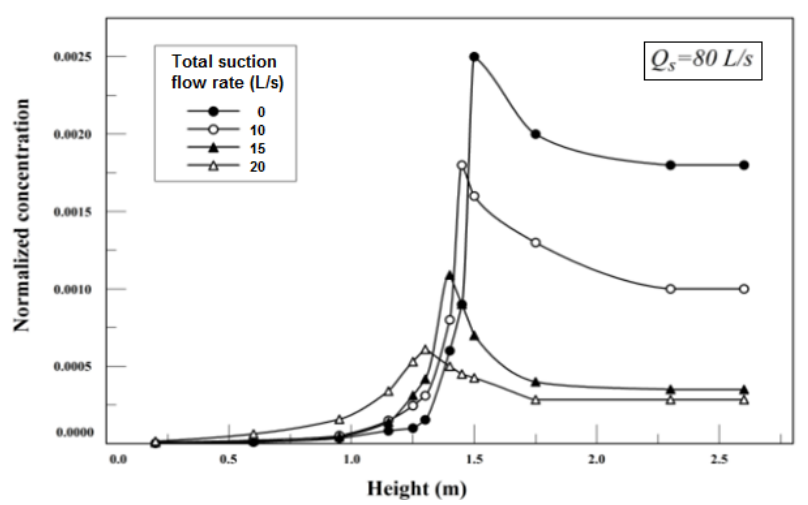

Fig. 2: Effect of the total CF flow rate per occupant when assisting DV on: a) the intake fraction of $1 \mu \mathrm{m}$ particles at the exposed occupant breathing level for different DV supply flow rates; b) the variation with height of the average normalized concentration of $1 \mu \mathrm{m}$ particles within the surrounding air for a DV supply flow rate of $80 \mathrm{~L} / \mathrm{s}$

\section{B. Assisting CPV by $C F$}

In opposition to the case of assisting DV by $\mathrm{CF}$, the goal of assisting CPV with $\mathrm{CF}$ was to decrease the strength of the thermal plumes. In the studied $C P V$ system, each occupant was supplied with fresh air $\left(10 \mathrm{~L} / \mathrm{s}\right.$ at $\left.16^{\circ} \mathrm{C}\right)$ downward from a ceiling single nozzle centered within a peripheral diffuser delivering recirculated air $(35 \mathrm{~L} / \mathrm{s}$ at $16^{\circ} \mathrm{C}$ ) at $45^{\circ}$ angle resulting in a canopy around the occupant (fig. 3). Figures 3 and 4 represent a comparison, between the standalone CPV system and the CPV system assisted by $\mathrm{CF}$ sucking air at $10 \mathrm{~L} / \mathrm{s}$, of the velocity and 
temperature fields respectively in the two-station office space at the symmetry plane. As the rising thermal plumes oppose the delivery of the CPV jets, the efficiency of the CPV system can be increased by suppressing the human convective plumes (Figs. 3-4).

With the increase of $\mathrm{CF}$ flow rate, the $C P V$ jet momentum is strengthened reaching the breathing level more effectively (Fig. 3). Figure 5 represents the effect of the total CF flow rate per occupant when assisting CPV on the intake fraction of $1 \mu \mathrm{m}$ particles at the exposed occupant breathing level.

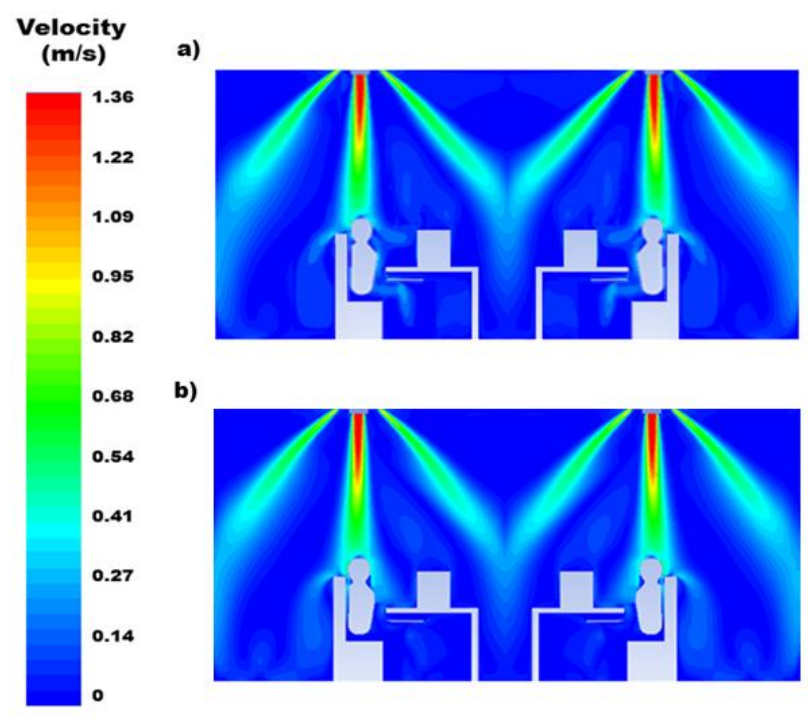

Fig. 3: Representation of the velocity field in the two-station office space at the symmetry plane for: a) Standalone CPV system; b) CPV assisted by CF sucking air at 10L/s.

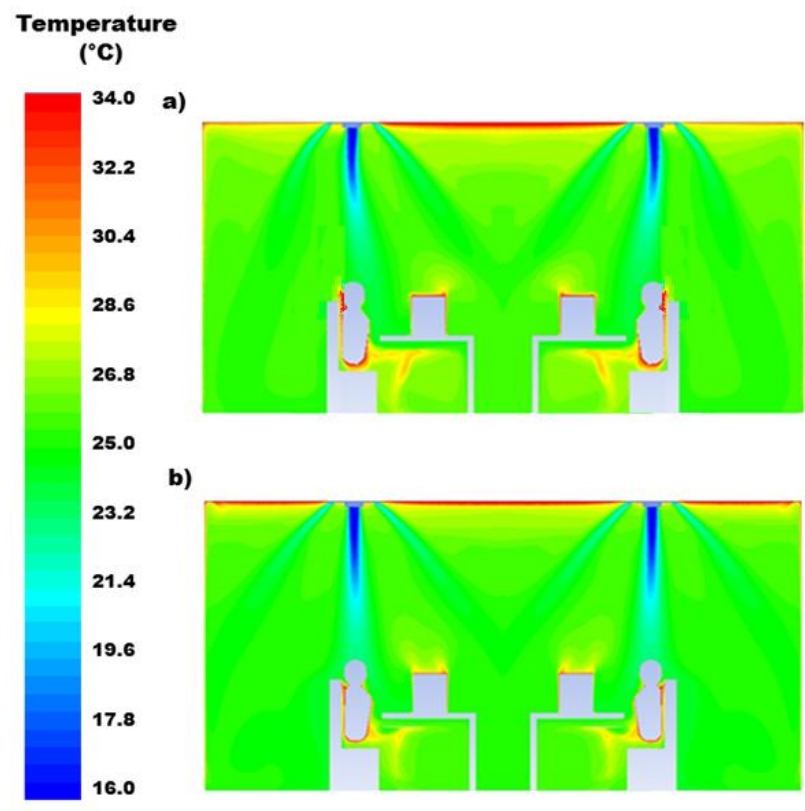

Fig. 4: Representation of the temperature in the two-station office space at the symmetry plane for: a) Standalone CPV system; b)

$\mathrm{CPV}$ assisted by $\mathrm{CF}$ sucking air at $10 \mathrm{~L} / \mathrm{s}$.
For $\mathrm{CF}$ flow rate flow between 0 and $10 \mathrm{~L} / \mathrm{s}$, the presence of $\mathrm{CF}$ reduced the intake fraction resulting in significant enhancement of the CPV performance (Fig. 5). Figure 6 represents the concentration of $1 \mu \mathrm{m}$ particles in the twostation office space at the symmetry plane for standalone CPV system compared to CPV assisted by CF sucking air at $10 \mathrm{~L} / \mathrm{s}$.

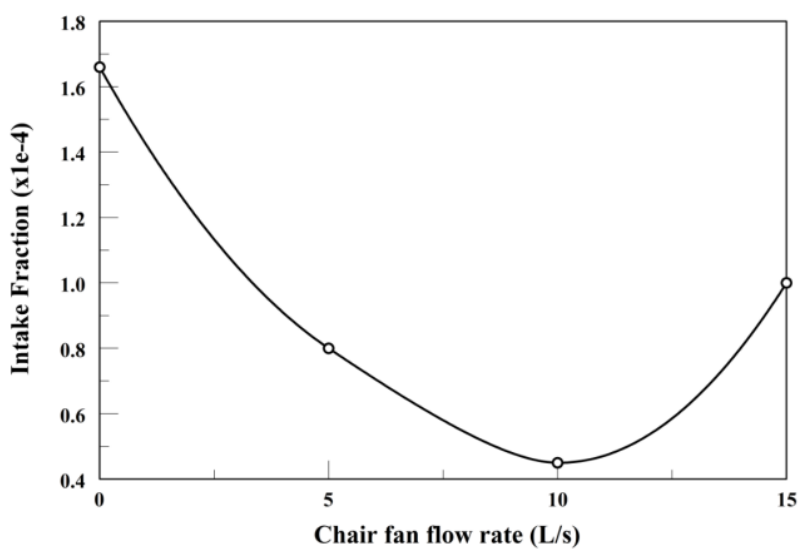

Fig. 5: Effect of the total CF flow rate per occupant when assisting CPV on the intake fraction of $1 \mu \mathrm{m}$ particles at the exposed occupant breathing level.

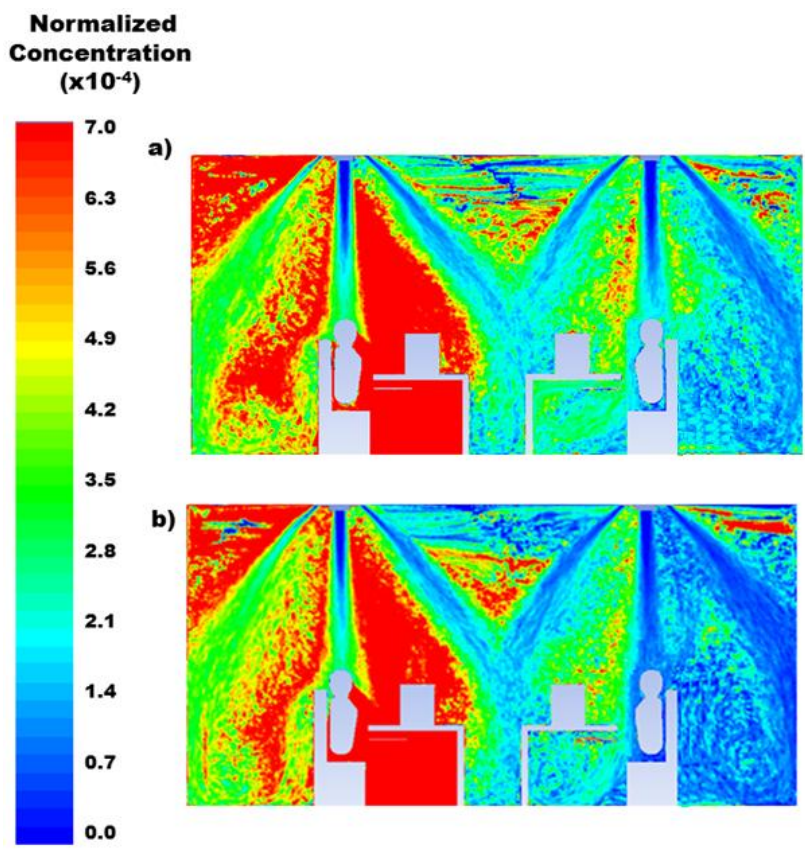

Fig. 6: Representation of the concentration of $1 \mu \mathrm{m}$ particles in the two-station office space at the symmetry plane for: a) Standalone CPV system; b) CPV assisted by CF sucking air at $10 \mathrm{~L} / \mathrm{s}$.

The $C F$ decreased particle transport between the two stations and increased the exposed person protection via facilitating clean air delivery to the breathing level of the occupants (Fig. 6). Nevertheless, CF operation resulted in more air mixing within the canopy created by the diffusers of the CPV system. This enhanced contaminant mixing close to the breathing level tending to increase the 
intake fraction. The two opposing effects of CF operation resulted in an optimal total $\mathrm{CF}$ flow rate per occupant of approximately $10 \mathrm{~L} / \mathrm{s}$ insuring a minimal IF (Fig. 6).

Therefore, the single nozzle fresh air flow rate can be significantly reduced when $\mathrm{CPV}$ is assisted by $\mathrm{CF}$ to obtain equivalent IAQ compared to the standalone $\mathrm{CPV}$ system reducing largely the energy consumption.

\section{Conclusion}

In this work, the ability of vertical and horizontal localized ventilation systems in reducing cross-contamination between occupants in office spaces was investigated and their assistance with $\mathrm{CF}$ was proposed to enhance their performance in terms of IAQ and energy consumption.

A CFD model was developed to investigate the effect of the CF operation on DV and CPV performance in respect with reduced cross-contamination between occupants.

Assisting different types of localized ventilation by $\mathrm{CF}$ enhanced their performance by proper control of the rising thermal plumes strength in correspondence with the type of localized system used. For instance, when assisting DV, for which air motion is triggered by buoyancy effects, the $\mathrm{CF}$ were operated to increase the strength of rising thermal plumes by blowing air upwards thus favoring the removal of contaminants and exposed occupant protection from disease transmission. On the other hand, when aiding CPV the $\mathrm{CF}$ were used to suppress the occupant convective plumes opposing the fresh air delivery by sucking air downward therefore facilitating the propagation of the downward CPV jet.

The total CF flow rate per occupant was optimized for both cases to insure high IAQ resulting in significant energy savings. It was found that the optimal total CF flow rate per occupant was $14 \mathrm{~L} / \mathrm{s}$ when assisting DV while it was $10 \mathrm{~L} / \mathrm{s}$ when aiding CPV.

\section{Acknowledgement}

The authors would like to thank the Lebanese National Council for Scientific Research (CNRS) for their financial support. The support of the University Research Board (URB) at the American University of Beirut is also acknowledged as well as the Shammas PhD Fellowship.

\section{References}

[1] Sexton K, Ramachandran G, Pratt C, Mongin J, Stock H, and Morandi T, Comparison of personal, indoor, and outdoor exposures to hazardous air pollutants in three urban communities. Environ. Sci. Technol. 2004; 38: 423-430

[2] Shoeib M, Harner T, Wilford H, Jones C, Zhu J. Perfluorinated sulfonamides in indoor and outdoor air and indoor dust: occurrence, partitioning, and human exposure. Environ. Sci. Technol. 2005; 39: 6599-6606

[3] Sandle, T. People in Cleanrooms: Understanding and Monitoring the Personnel Factor| IVT. Hand, 10, 100-000.

[4] Nicas M, Nazaroff WW, Hubbard A. Toward understanding the risk of secondary airborne infection: emission of respirable pathogens. Journal of occupational and environmental hygiene 2005; 2(3): 143-154.
[5] IPD Occupiers. Efficiency Standards for Office Space, A report to Office of Government Commerce, http://ns.kinnarps.tm/Countries/UK/PS\%20minisite/Agree ment/Efficiency _Standards _for_Office_Space.pdf, November 2007.

[6] Melikov AK. Personalized ventilation. Indoor Air 2004; 14(s7): 157-167.

[7] Olli S. Ventilation Strategies for Good Indoor Air Quality and Energy Efficiency. International Journal of Ventilation 2008; 6(4): 297-306.

[8] Makhoul, A, Ghali K, Ghaddar N, Thermal comfort and energy performance of a low-mixing ceiling-mounted personalized ventilator system, Building and Environment 2013; 60: 126-136.

[9] Melikov AK. Human body micro-environment: the benefits of controlling airflow interaction. Build Environ 2015; doi:10.1016/j.buildenv.2015.04.010.

[10] Chen FZ, Yu S, Lai A. Modeling particle distribution and deposition in indoor environments with a new drift-flux model. Atmospheric Environment 2006; 40: 357-367.

[11] ANSYS Software: ANSYS Inc. http://www.ansys.com/

[12] Zhu S, Kato S, Yang JH. Study on transport characteristics of saliva droplets produced by coughing in a calm indoor environment. Building and Environment 2006; 41(12): 1691-702.

[13] Zhang Z, Chen Q. Comparison of the Eulerian and Lagrangian methods for predicting particle transport in enclosed spaces. Atmospheric Environment 2007; 41: 5236-5248.

[14] Wang M, Lin CH, Chen Q. Advanced turbulence models for predicting particle transport in enclosed environments. Building and Environment 2012; 47: 40-49.

[15] Makhoul A, Ghali K, Ghaddar N. Investigation of Particle Transport in Offices Equipped with Ceiling-Mounted Personalized Ventilators. Building and Environment 2013; 63: 97-107.

[16] Zhang Z, Chen Q. Experimental measurements and numerical simulations of particle transport and distribution in ventilated rooms. Atmospheric Environment 2006; 40(18): 3396-3408.

[17] Habchi C, Ghali K, Nesreen Ghaddar, Walid Chakroun, Sorour Alotaibi. Ceiling Personalized Ventilation Combined with Desk Fans for Reduced Direct and Indirect Cross-Contamination and Efficient use of an Office Space. Energy Conversion and Management, 2016; Volume 111, Pages 158-73.

[18] Makhoul A, Ghali K, Ghaddar N. Desk fans for the control of the convection flow around occupants using ceiling mounted personalized ventilation. Building and Environment 2013; 59: 336-348.

[19] Li X, Niu J, Gao N. Spatial distribution of human respiratory droplet residuals and exposure risk for the cooccupant under different ventilation methods. HVAC\&R Research 2011; 17: 432-445.

[20] Braaten DA. Wind tunnel experiments of large particle reentrainment-deposition and development of large particle scaling parameters. Aerosol Science and Technology 1994; 21(2):157-169.

[21] Makhoul A, Ghali K, Ghaddar N. The energy saving potential and the associated thermal comfort of displacement ventilation systems assisted by personalized ventilation. Indoor Built Environment 2013; 22: 508-519. 